Received 00th January 20xx, Accepted 00th January 20xx DOI: $10.1039 / x 0 x \times 00000 x$

\title{
Effect of molecular weight on the EUV-printability of main chain scission type polymers
}

\author{
Ashish Rathore, ${ }^{* a, b}$ Ivan Pollentier ${ }^{a}$, Harpreet Singh ${ }^{c}$, Roberto Fallica ${ }^{a}$, Danilo De Simone ${ }^{a}$, Stefan \\ De Gendt ${ }^{\mathrm{a}, \mathrm{b}}$ \\ aIMEC, Kapeldreef 75, 3001 Leuven, BE \\ ${ }^{b} K U$ Leuven Department of Chemistry, Celestijnenlaan 200, 3001 Leuven, BE \\ ${ }^{c}$ Centre for nanoscience and nanotechnology, Panjab University, 160014 Chandigarh, IN
}

\begin{abstract}
Unclear light-matter interactions in the Extreme UV lithography (EUVL) process cause the sub-optimal performance of the current photoresist platforms. Furthermore, adopting conventional chemically amplified resist (CAR) is problematic in EUVL because of the exacerbated stochastic effects. Therefore, alternative platforms with simpler chemistry, such as the main chain scission (MCS)-type photoresist, can pave the way for efficient printability at higher resolutions. In this work, fundamental analysis of a simple MCS-type photoresist - Polymethyl methacrylate (PMMA), is studied and correlated with the parameters of EUV-patterning (called litho-parameters). Contrast curve analysis with different solvents shows MIBKIPA(1:3) mixture as the best developer for EUV-patterning of PMMA. Higher Mw (950K) shows the best EUV-printability compared to the lower MW (15K, 120K, and 600K) PMMA for $50 \mathrm{~nm}$ dense line-space (L/S). To understand the effect of polymer properties on printability, desorption and Gel Permeation Chromatography (GPC) studies are conducted, which show that the higher MW PMMA provides better litho-parameters (higher MCS - to - side-chain scission (SCS) ratio, higher GPC peak shift, and lower GPC peak overlap after EUV-exposure) compared to the lower MW PMMA. Additionally, it is found that the PMMA chains require minimum energy of $9 \mathrm{eV}$ to induce chemical reactions in the film. The main hypothesis obtained from this study is that for MCS-type EUV resists, higher Mw material provides better printability than the lower Mw material. This is further validated on an engineered MCS-copolymer system. Finally, it is observed that the inhomogeneous dosedeposition during EUV-exposures might hamper the printability of the MCS-type resist.
\end{abstract}

\section{Introduction}

EUV lithography (EUVL) has recently been introduced for high volume manufacturing (HVM) in the semiconductor industry. ${ }^{1}$ This process can print at sub- $5 \mathrm{~nm}$ technology nodes and is the successor of the conventional lithography (for eg. - Deep Ultraviolet Lithography, DUVL). However, to effectively shift towards EUVL, several challenges need to be overcome. Firstly, the energy of the source EUV-radiation $(91.6 \mathrm{eV})$ is much higher than the radiation source used, for example, in DUV (approx. 6 $\mathrm{eV})$. This changes the light-matter interactions in the exposed photoresist from excitation chemistry (in DUVL) to radiation chemistry (in EUVL). ${ }^{2-4}$ That is, low energy photons of DUV can only cause excitation chemistry, whereas, high energy EUVphotons cause radiation chemistry and generate a cascade of secondary electrons in the photoresist film. These secondary

\footnotetext{
a. Address here.

b. Address here.

c. Address here.

† Footnotes relating to the title and/or authors should appear here.

Electronic Supplementary Information (ESI) available: [details of any supplementary information available should be included here]. See DOI: 10.1039/x0xx00000x
}

electrons then interact with the photoresist molecules through elastic and inelastic scattering events that induce chemical reactions to yield a solubility switch in the exposed region of the photoresist. The details of these interactions are currently unclear and cause the existing photoresist platforms to perform sub-optimally for EUVL.

Another major problem associated with the current EUVphotoresist platforms is the Resolution-Line edge roughnessSensitivity (RLS) trade-off. ${ }^{5,6}$ Resolution is the smallest feature that can be printed using a photoresist; Line-edge-roughness (LER) is the deviation from an ideal smooth shape and sensitivity is the smallest exposure dose needed to print at that resolution. It is proving impossible to improve two of the above parameters without exacerbating the third (and hence the trade-off). The third major problem is called stochastic failures. ${ }^{7-9}$ It is the nanofailures caused by the photon shot noise ${ }^{10}$ and local chemical variability in the photoresist during EUVL. These nanofailures result in defects such as broken lines, nano-bridges and increases the LER of the patterns. Only by understanding the fundamental chemistry involved in the EUVL process, it may be possible to control RLS trade-off and stochastic failures. There 
have already been some strides in comprehending the chemistry of the EUVL process. ${ }^{4,5,11-15}$ However, most of these studies focus on the photoresist system as a whole, which makes understanding fundamentals a daunting task.

Furthermore, adopting conventional chemically amplified resists (CARs) for EUVL is proving tough, as they pattern through an acid diffusion process, and controlling this diffusion process is difficult. The uncontrolled diffusion increases the LER and causes a lot of stochastic defects in the patterns. ${ }^{15}$ The complex chemistry of CARs, containing multiple components, also plays a significant role in the problems associated with this platform. ${ }^{16}$ Therefore, alternatively, non-CAR photoresist platforms, with patterning mechanisms other than the acid diffusion, are being developed for EUVL. One such type is the main chain scission (MCS) photoresist platform. ${ }^{17,18}$ These photoresists pattern through direct scission in their polymer chain when exposed to EUV-radiation, simplifying the lithochemistry, which can help in reducing the LER and stochastic defects. However, further development and optimization is needed in the MCS photoresist platform to be workable at sub$5 \mathrm{~nm}$ tech nodes.

The aim of this work is to simplify the problem by studying a model MCS-type system (Polymethylmethacrylate, PMMA) and identify important litho-parameters. The results obtained on the simple system are then tested on an engineered copolymer system for validation. This study is essential in the optimization and development of novel MCS-type photoresists with high EUV-printability.

\section{Experimental details}

\subsection{Materials}

15,000 (15K), 120K and 350K Da Mw PMMA are obtained from Sigma Aldrich, whereas, 600K and 950K Da Mw PMMA are obtained from Allresist $\mathrm{GmbH}$. Engineered MCS-type copolymer systems, with the same chemistry but different $\mathrm{Mw}$, is obtained from Zeon corporation and tested for EUVL as received. The structure of these systems is similar to ZEP-520A, ${ }^{18}$ consisting of 1:1 ratio of monomers - chloromethyl acrylate and methyl styrene. Furthermore, solvents used for development step and other reagents are obtained from Sigma-Aldrich.

\subsection{Methodology}

2.2.1 Contrast curve analysis and patterning with Imec's ASML NXE3300B EUV-scanner

Contrast curve analysis is performed on Imec's outgas tool ${ }^{19}$ mounted with a EUV-source. The photoresist material is coated on a $200 \mathrm{~mm}$ Si wafer and film thickness and uniformity is checked with an ellipsometer. Multiple points are exposed to a variable EUV-dose. The development step is carried on the exposed wafer with different developers to find the best developer solvent and dose to clear $\left(E_{0}\right)$ for the material. Patterning experiments are conducted on Imec's ASML NXE3300B EUV-scanner to check the printability of line/space
(L/S) patterns of the dense pitch of $100 \mathrm{~nm}$ (for PMMA) and 36 $\mathrm{nm}$ (for copolymer system). Scanning electron microscopy (SEM) analysis is done on the Hitachi Critical dimension (CD)SEM systems.

2.2.2 EUV and low electron energy (LEE)-induced desorption EUV and LEE-induced desorption of volatile species from the photoresist film are studied by the Residual Gas Analyzer (RGA) installed in Imec's outgas tool. ${ }^{19}$ Upon exposing the spin-coated resist material to radiation (EUV and LEEs), the photoresist film undergoes chemical reactions, which results in the desorption of certain fragmental species. These species are picked up by the mass spectrometer and a fragmentation mass spectrum is obtained. The spectrum is then compared with the existing database (eg. NIST Chemistry Webbook ${ }^{20}$ ) to identify the desorbed species. This is useful to understand the EUV and LEEs-induced chemical interactions in the film. The detailed setup and the description of the tool can be obtained from [21].

\subsubsection{Gel permeation chromatography (GPC)}

GPC works on the principle of size exclusion ${ }^{22}$ and is used to measure the changes in the polymer property (Mw) of MCStype material after EUV-exposure. 2 sets of $300 \mathrm{~mm}$ Si wafers are spin-coated with the photoresist. One set is stripped with Tetrahydrofuran (THF) solvent right after coating, whereas the other set is exposed in Imec's ASML NXE3300B EUV-scanner (full field flood exposure) and stripped. The Mw of unexposed (reference) and exposed samples are analyzed with the Shimadzu-GPC system connected with a refractive index detector (RID-20A).

\subsubsection{CHARIOT simulation tool}

A Monte Carlo simulation tool, called CHARIOT, ${ }^{23}$ is used to understand the effects of the EUV-exposure on polymer films. It uses a physical model to track the movements of low energy electrons and holes in the resist film, to provide an understanding of the charge distribution throughout the film. This simulation tool can help explain some of the effects seen during the experimentations. The full description of the tool can be found in [23].

\section{Results and discussion}

\subsection{Contrast curve analysis and EUV-printability of PMMA}

Contrast curve analysis is carried on PMMA resist to find the best exposure conditions. Figure 1 shows the results of $600 \mathrm{~K}$ Mw PMMA for different developer solvents. These solvents are selected based on their differences in the polarity - from nonpolar to polar. Some of these developer solvents have already been reported for PMMA patterning for $\mathrm{EBL}^{24,25}$ and are now tested for EUV-exposure. As seen from figure 1, MIBK (a strong nonpolar solvent) readily dissolves PMMA exposed even at a very low dose, which is not ideal for litho-application. On the other hand, both IPA and $\mathrm{H}_{2} \mathrm{O}$-IPA (7:3) mixture (polar solvents) show low solubility to EUV-exposed PMMA, which is again nonideal for litho-application. MIBK-IPA (1:3) mixture provides the 
best contrast with a nominal dose-to-clear $\left(E_{0}\right)$ value of 25 $\mathrm{mJ} / \mathrm{cm}^{2}$. This confirms that MIBK-IPA (1:3) mixture is an optimal solvent for EUV patterning of PMMA, the same as E-beam patterning. Furthermore, it is observed that the film thickness (FT) starts to increase again at a high EUV-dose of $600 \mathrm{~mJ} / \mathrm{cm}^{2}$ and beyond, as PMMA chains start to crosslink. This is also identified by the visual observation that the material becomes insoluble to any of the developers when exposed above that dose.

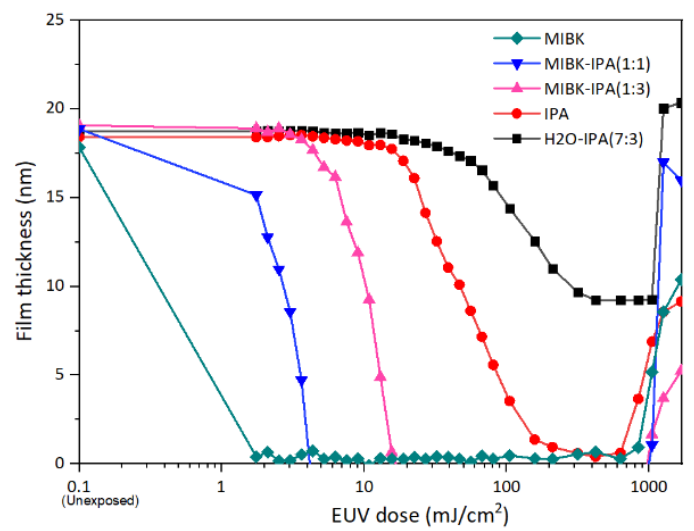

Figure 1 Contrast curve analysis of 600K MW PMMA for variable EUVdose and developer solvents.

After the contrast curve analysis, EUV-printability of different Mw PMMA is checked for $50 \mathrm{~nm}$ dense L/S patterns in Imec's ASML NXE3300B scanner. The patterning images and the SEM data are provided in table 1. 15K Mw PMMA shows no resolution at $50 \mathrm{~nm}$ dense L/S. However, as the $\mathrm{Mw}$ is increased to $120 \mathrm{~K}$, PMMA starts to print but with a high LER value. Upon further increase of $\mathrm{Mw}$ to $600 \mathrm{~K} \mathrm{Mw} \mathrm{PMMA}$, the contrast and the LER of the patterns improve, and the 950K Mw PMMA shows the best patterning performance with good image contrast and lowest LER among all the different Mw materials.

The complete criteria used to determine the EUV-printability performance of different $\mathrm{Mw}$ material is provided in section S2 of the supplementary information.

Table 1 SEM images of different Mw PMMA for $50 \mathrm{~nm}$ dense line-space (L/S) patterns
EUV-patterning results show that the Mw of starting material is an important factor influencing the printability of MCS-type photoresist. To further characterize this, fundamental studies are carried on different Mw PMMA materials and the results are provided in the following sections.

\subsection{Ester decarboxylation as a measure of scission in PMMA chains}

It has been reported in the literature that exposure to high energy radiation (electrons and $\mathrm{X}$-ray) causes scission of the PMMA chains resulting in the ester decarboxylation process. ${ }^{26-}$ ${ }^{29}$ Ester decarboxylation can be due to two different scission mechanism occurring in the chains. The first one is the mainchain scission (MCS) type, where the polymer backbone fragments and produces methyl methacrylate as the desorption species, as shown in figure 2(a). The second one is the side-chain scission (SCS) type, where the polymer backbone remains intact, and fragmentation happens only on the side chains, which produces methyl formate desorption species as shown in figure 2(b). It is also seen that the SCS can induce further fragmentation in the backbone and cause MCS, as shown in figure 2(c). The amount of these desorption species, methyl methacrylate, and methyl formate can be calculated by the RGA to determine the degree of MCS and SCS occurring in different Mw PMMA. The RGA results are provided in the next section.

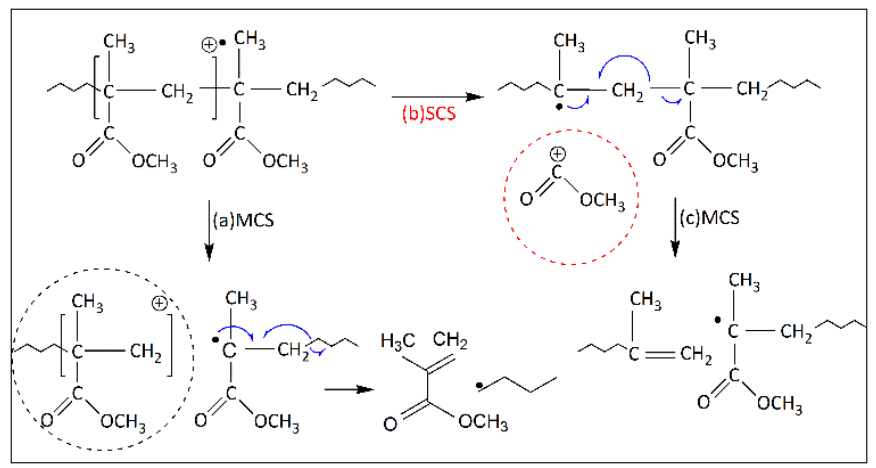

Figure 2 Simplified mechanism of radiation-induced (a) main chain scission (MCS) generating desorption species - methyl methacrylate (circled in black) and (b) side chain scission (SCS) generating the desorption species - methyl formate (circled in red) in PMMA chains, (c) SCS can further induce MCS in some chains. [adapted from ref. 28].

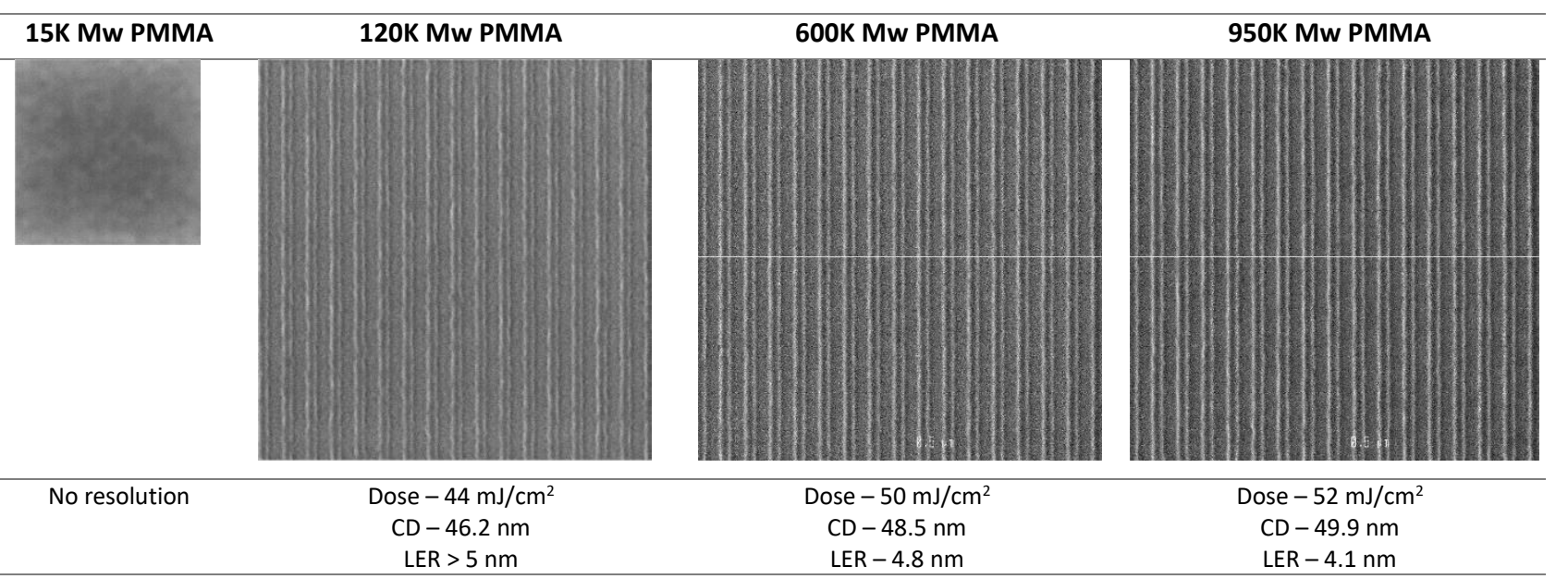

This journal is (C) The Royal Society of Chemistry 20xx 
3.3 Desorption and GPC studies to check the amount of MCS and SCS in different Mw PMMA

\subsubsection{EUV-induced desorption}

EUV-induced scission of the PMMA chains is studied by the RGA. Figure 3(a) shows the fragmentation mass spectrum of the outgassing species captured by the RGA from 600K Mw PMMA (film thickness (FT) $-40 \mathrm{~nm}$ ) when exposed to a EUV-dose of $40 \mathrm{~mJ} / \mathrm{cm}^{2}$. Upon comparing this spectrum with the database, it shows the desorption of the following major species - methyl methacrylate (amu-100), methyl formate (amu-60), carbon dioxide (amu- 44) and methanol (amu-32). The other peaks correspond to the further fragmentation of these larger species. For determining if MCS or SCS events occur, it is important to study the desorption peaks of the following two larger species. The first peak at the atomic mass unit (amu) 100 is methyl methacrylate, which is a product of main chain scission (MCS) in the PMMA chains. And the second peak at amu 60 is methyl formate, which is a product of side-chain scission (SCS) in PMMA (figure 3(b)).
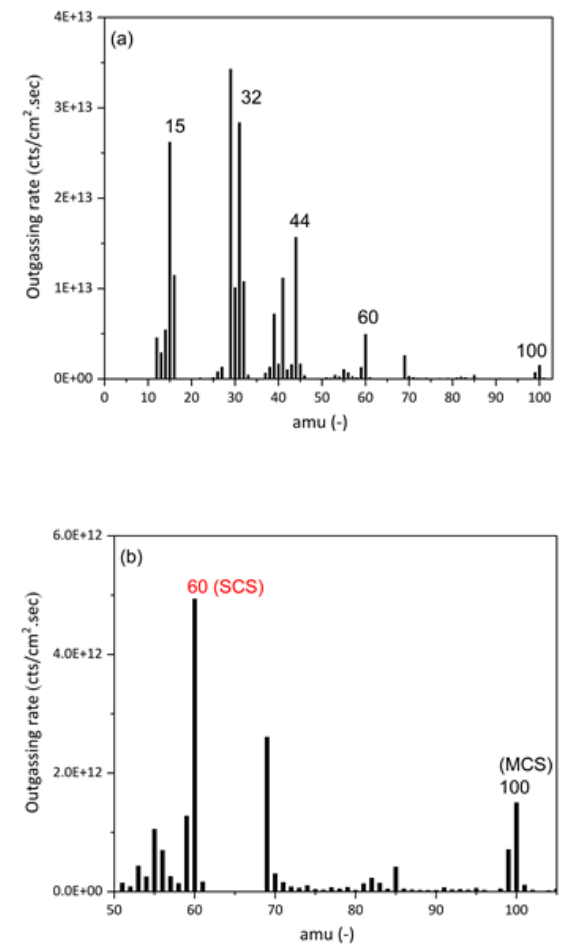

Figure 3 (a) Fragmental mass spectrum of 600K Mw PMMA when exposed to a EUV-dose of $40 \mathrm{~mJ} / \mathrm{cm}^{2}$ and (b) zoomed in version showing methyl methacrylate (amu-100) and methyl formate (amu-60) peak as a measure of MCS and SCS respectively.

The amount of desorption of the MCS and SCS fragments as a function of EUV-dose can be calculated from the outgassing rate of methyl methacrylate (amu 100) and methyl formate (amu $60)$. Figure $4(a)$ shows the RGA results for 600K Mw PMMA. Upon EUV-exposure, the amount of MCS fragments captured by the RGA is much lower (approx. 5 times) as compared to the amount of SCS. Additionally, the intensities of MCS and SCS fragments drop at a very high dose, which is because of the crosslinking in the polymer chains as well as the saturation effect at high EUV-dose. Similarly, RGA can be used to calculate the outgassing rate of MCS and SCS-fragments in different $\mathrm{Mw}$ PMMA films (coated using the solution of similar densities of $\sim 0.99 \mathrm{~g} / \mathrm{cm}^{3}$ to achieve a final FT of $40 \mathrm{~nm}$ ). The results are shown in figure $4(\mathrm{~b})$.
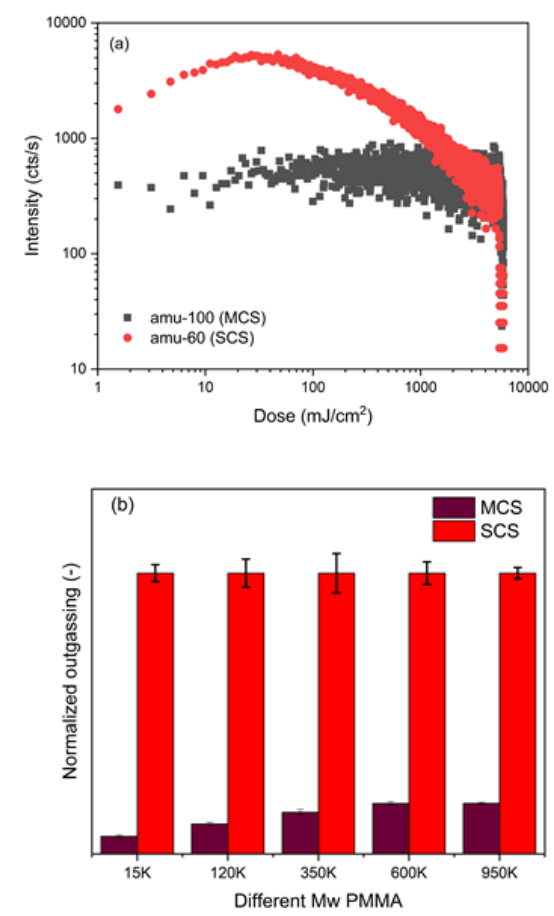

Figure 4 (a) Amount of EUV-induced MCS and SCS in 600K MW PMMA as a function of the EUV-dose and (b) Comparison of the amount of EUVinduced MCS and SCS in different MW PMMA.

RGA analysis of all the different Mw PMMA shows the detection of a higher number of SCS-fragments as compared to the MCSfragments. This can be explained by the reason that for producing an SCS-fragment, only one bond needs to be broken from the polymer, whereas for producing an MCS-fragment, two bonds in the polymer have to be broken. Moreover, the $\mathrm{O}=\mathrm{C}-\mathrm{C}$ bond of the side chain is easier to break compared to $\mathrm{C}$ $\mathrm{C}$ bonds of the main chain.

A ratio of MCS-to-SCS is calculated for different Mw PMMA by dividing the number of MCS and SCS-fragments captured by the RGA, and the values are provided in table 2 . It is seen that the MCS-to-SCS ratio is statistically similar for $350 \mathrm{~K}, 600 \mathrm{~K}$ and $950 \mathrm{~K}$ $\mathrm{Mw}$ PMMA. However, when considering 15K, 120K, and $600 \mathrm{~K} / 950 \mathrm{~K} \mathrm{Mw} \mathrm{PMMA}$, it is found that the ratio is proportional to the Mw of PMMA. For instance, the value of this ratio is approximately three times higher for 600K and 950K Mw PMMA than the $15 \mathrm{~K} \mathrm{Mw} \mathrm{PMMA.} \mathrm{This} \mathrm{suggests} \mathrm{that} \mathrm{the} \mathrm{higher} \mathrm{Mw}$ material might have a better tendency to undergo MCS than SCS. Therefore, high Mw material is better for MCS-type photoresists. Also, to further increase this ratio, it might be necessary to modulate the chemistry to favor MCS-type scissions over SCS-type scission in the polymer chains. 
Table 2 The ratio of main-chain scission (MCS) to side-chain scission (SCS) for different MW PMMA.

\begin{tabular}{cc}
\hline Different Mw PMMA & Value of MCS-to-SCS ratio \\
\hline 15K PMMA & $0.06 \pm 0.02$ \\
\hline 120K PMMA & $0.11 \pm 0.02$ \\
\hline 350K PMMA & $0.14 \pm 0.03$ \\
\hline 600K PMMA & $0.17 \pm 0.02$ \\
\hline 950K PMMA & $0.17 \pm 0.01$ \\
\hline
\end{tabular}

Please note that the amount of MCS and SCS-fragments captured by the RGA has a significant standard deviation. The reasons behind this are as follows. EUV-photons expose the whole cross-section of the film and cause MCS and SCS in the bulk. To be captured by the RGA, the fragments have to diffuse from the bulk to the surface. The diffusion length of the smaller fragments (amu-60) is higher than the larger fragments (amu100) and hence more SCS-fragments are likely to be captured by the RGA. Also, during the diffusion process, the MCSfragments can undergo further fragmentation by the incoming photons and secondary electrons present in the film. Thus, the amounts captured may vary for different experimental trials. But even with the substantial standard deviations, RGA provides a good insight into the type of EUV-induced scission occurring in the polymer film.

\subsubsection{LEE-induced desorption}

LEEs are important in EUVL, as the cascade of low energy secondary electrons are responsible for most of the lithochemistry induced in the photoresist film. Therefore, LEEinduced desorption studies are conducted to understand their effect on PMMA.

Like EUV-radiation, LEEs also induce MCS and SCS scission in PMMA. Figure 5(a) shows the LEE-induced chemical yield of MCS (methyl methacrylate; amu - 100) and SCS-products (methyl formate; amu -60 and $\mathrm{CO}_{2}$; amu -44) observed in $600 \mathrm{~K}$ Mw PMMA. It is observed that the activation energy seems to be around $9 \mathrm{eV}$ for PMMA. The activation energy here refers to the minimum energy required to sufficiently induce bond cleavages in the PMMA polymer chains to produce MCS and SCS-fragments that are captured by the RGA. Additionally, the amount of LEE-induced MCS and SCS is calculated by the RGA and is shown in figure 5(b). It shows that the number of MCSfragments for 600K Mw PMMA is almost 2.5 times higher than the 15K Mw PMMA. Whereas, the number of SCS-fragments was only 1.3 times higher, as captured by the RGA. This confirms the previous observation that the higher Mw PMMA has a higher tendency to undergo MCS than the lower Mw PMMA.

Furthermore, figure 5(b) shows that the amount of MCS is higher than SCS for LEEs, which is contradictory to what is seen in the EUV-exposed PMMA material. The following are the reasons that can cause the detection of a higher number of MCS-fragments for LEEs.
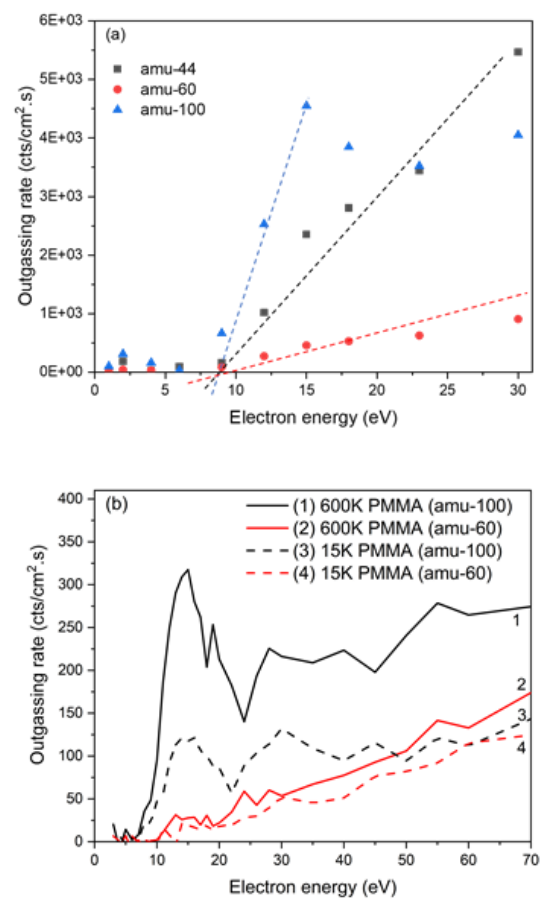

Figure 5 (a) Amount of EUV-induced MCS and SCS in 600K MW PMMA as a function of the EUV-dose and (b) Comparison of the amount of EUVinduced MCS and SCS in different MW PMMA.

Firstly, the theoretical inelastic mean free path of $9-30 \mathrm{eV}$ electrons is in the range of $3-10 \mathrm{~nm},{ }^{30}$ therefore, most of the reactions for the LEEs are happening only on the top surface of the film, unlike the EUV-radiation, which exposes the whole cross-section. This means that the larger fragments generating in the film for LEEs have a higher chance of getting captured by the RGA. Also, because the MCS-fragments have to diffuse to a much smaller distance before getting captured, the probability of further fragmentation is vastly reduced. Furthermore, the electron energy distribution in the resist film is different for EUV and LEE-exposure. And figure 5(b) shows that as the energy of the incident electrons is reduced, the MCS-to-SCS ratio increases. This can mean that the electrons with very low energies (and with high mean free paths ${ }^{30}$ ), can diffuse to a larger distance during the thermalization process in the film and can cause multiple scissions in the backbone, thus increasing the probability of MCS. Finally, it is known that the electroninduced SCS can also induce further MCS in the PMMA chains, ${ }^{28,31}$ and hence some of the SCS cause a further increase in the intensity of the MCS fragments.

\subsubsection{GPC characterization}

Different Mw PMMA is exposed to an EUV-dose of $30 \mathrm{~mJ} / \mathrm{cm}^{2}$ and GPC characterization is done to confirm the amount of MCS in the different Mw PMMA. The GPC results are provided in figure 6(a), which shows the intensity of the unexposed (reference) and exposed peak versus the retention time for $15 \mathrm{~K}$ 
and 950K Mw PMMA. Complete data is provided in section S4 of the supplementary information.

Based on this, two important litho-parameters have been identified to relate the changes in the polymer properties to the patterning results (figure $6(\mathrm{~b})$ ).
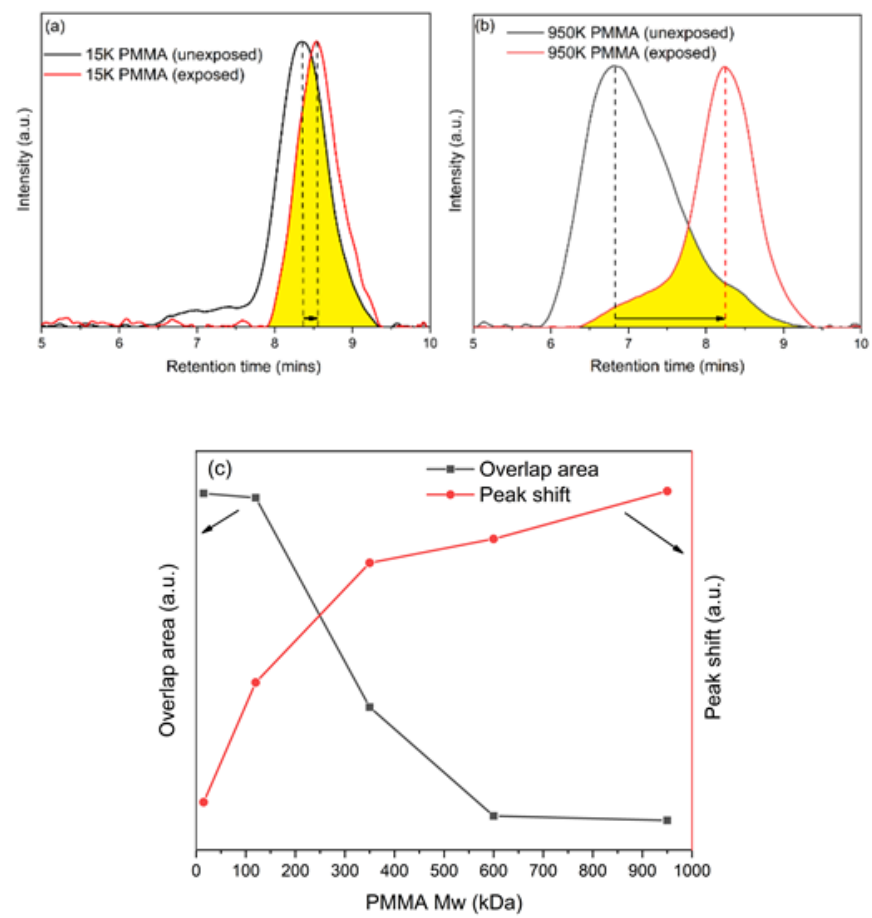

Figure 6 GPC analysis - comparison of the Mw peak shift and overlapping area in (a) $15 \mathrm{~K}$ and (b) $950 \mathrm{~K}$ MW PMMA before and after EUV-exposure at a dose of $30 \mathrm{~mJ} / \mathrm{cm}^{2}$. (c) The trend of the overlapping area and delta retention time (peak shift) in different MW PMMA after EUV-exposure.

The first important litho-parameter is the Mw peak shift between the reference and the exposed PMMA material. The amount of peak shift is found to be proportional to the Mw of the PMMA material, i.e., it follows the trend: $15 \mathrm{~K}<120 \mathrm{~K}<350 \mathrm{~K}$ $<600 \mathrm{~K}<950 \mathrm{~K}$ Mw PMMA (figure6(b)). This shift is good for MCS-type photoresist material because the higher the shift between the unexposed and exposed material, the higher the contrast between exposed and unexposed resist during patterning. This was confirmed by the contrast curve analysis of $120 \mathrm{~K}$ and 950K Mw PMMA for the same developer solvent (refer to section S5 of the supplementary information), which shows that the 950K, indeed, has a better chemical contrast compared to $120 \mathrm{~K} \mathrm{Mw}$ PMMA.

The second important litho-parameter is the overlapping area between the unexposed and exposed peak. The peak overlap follows the trend: $15 \mathrm{~K}>120 \mathrm{~K}>350 \mathrm{~K}>600 \mathrm{~K}>950 \mathrm{~K} \mathrm{Mw}$ PMMA (figure 6(b)). The overlapping area should be minimum for an MCS-type photoresist system, as this means the chain fragments are of different lengths for unexposed and exposed material, which ensures a better solvent development step during the lithography process.
Of all the materials, 950K Mw PMMA shows the best performance for both the litho-parameters explained above. Therefore, GPC analysis supports the EUV-patterning and desorption studies and provides additional insights as to why higher Mw starting material has a better EUV-printability compared to the lower Mw material.

Finally, it is noticeable for higher Mw PMMA (in figure 6(a)) that the GPC curve of exposed PMMA shows a bimodal distribution (shouldering in the high Mw region) after EUV-exposure. And this effect is only observed in EUV-exposures and not in the case of E-beam exposures, ${ }^{32}$ in which the exposed peak remains homogenous. One of the reasons for the peak shouldering could be the crosslinking in the polymer fragments after EUV exposure (although the cross-linking should be minimum at the applied low EUV-dose of $30 \mathrm{~mJ} / \mathrm{cm}^{2}$ ). A more likely reason can be the differences in the exposure conditions of EUV-radiation versus the high energy E-beam radiation. High energy E-beam causes a homogenous exposure throughout the resist film (because of the ultra-high energies used) which can result in homogenous fragmentation of the polymer chains. ${ }^{33,34}$ Whereas EUV-radiation causes inhomogeneous exposure in the film, that is, the top layer receives a higher dose compared to the bottom layer. This can result in insufficient exposure of some of the polymer chains and hence the formation of a shoulder in the exposed peak at the high Mw region. This effect is checked through a Monte Carlo study with the CHARIOT simulation tool in section 3.5 .

\subsection{Main hypothesis obtained from the analysis of different Mw PMMA}

From the patterning and fundamental analysis of PMMA, it is found that for an MCS-type photoresist, higher Mw starting material show better EUV-printability than the lower $\mathrm{Mw}$ material. The main reason for this is found to be the higher chemical contrast and better development process obtained after EUV-exposure for higher Mw material. Also, higher $\mathrm{Mw}$ material shows a higher amount of MCS-to-SCS ratio compared to lower Mw material. Therefore, it is hypothesized that for the MCS-type photoresist for EUVL, it is important to have a high Mw starting material.

The next step is to validate this understanding on an engineered copolymer MCS-type EUV-photoresist obtained from Zeon corporation. As mentioned in the material section, this system is similar to ZEP520A resist. The electronegative $\mathrm{Cl}$ atom on the polymer backbone is expected to increase the probability of MCS in the polymer chain when exposed to EUV-light. The presence of $\mathrm{Cl}$-atom also makes the material more sensitive compared to PMMA. It prints via main chain scission of the polymer backbone, similar to PMMA, but with higher sensitivity and resolution for EUV. ${ }^{35,36}$ The results are provided in the section below.

\subsection{Validation with the copolymer system}

Different $\mathrm{Mw}$ of the copolymer system are checked for their EUV-printability at $18 \mathrm{~nm}$ dense $\mathrm{L} / \mathrm{S}$, and the results are 
provided in table 3 . System 1 , with the lowest Mw of $20 \mathrm{~K}$, shows no printability. However, as the $\mathrm{Mw}$ of the starting material is increased to $50 \mathrm{~K}$ and $70 \mathrm{~K}$, the patterning performance improves and the photoresist system with the highest $\mathrm{Mw}$ of $350 \mathrm{~K}$ (system 4) shows the best EUV-printability. This validates that similar to PMMA, the highest Mw copolymer system shows the best EUV-printability.

Table 3 EUV-printability of different Mw MCS-copolymer system at 18 $n m$ dense $L / S$

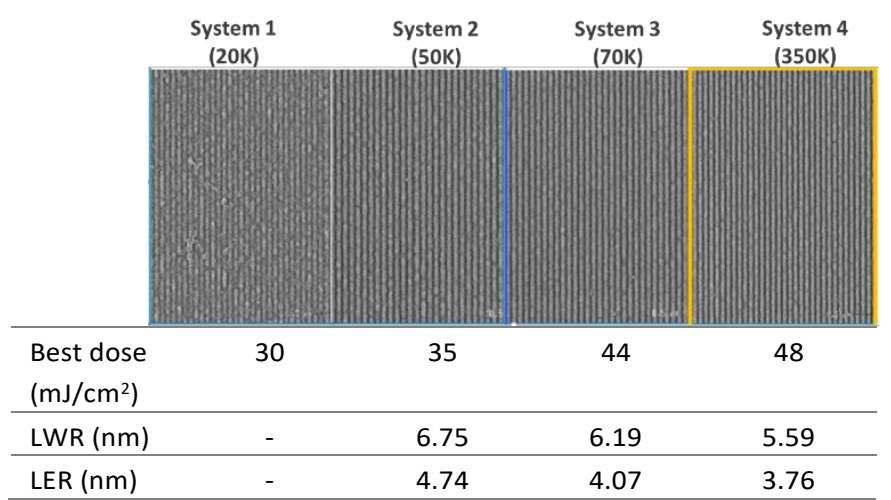

As a next step, system $3(\mathrm{Mw}-70 \mathrm{~K})$ is used to correlate the aforementioned litho-parameters with the EUV-printability of the copolymer system. To find the most optimum litho-parameters (namely, peak shift - evidencing fragmentation of the polymer chain and, peak overlap - evidencing the differences in the polymer chain lengths between the unexposed and exposed part), system 3 is exposed to a variable EUV-dose and changes in the polymer properties are analyzed by the GPC tool. GPC results are shown in figure 7 (a). From the GPC results, it was found that the dose of 30 and $50 \mathrm{~mJ} / \mathrm{cm}^{2}$ could not provide enough peak-shift between unexposed and exposed resist to have sufficient contrast. And the most suitable litho-parameters were achieved only at a high EUV-dose of $100 \mathrm{~mJ} / \mathrm{cm}^{2}$.
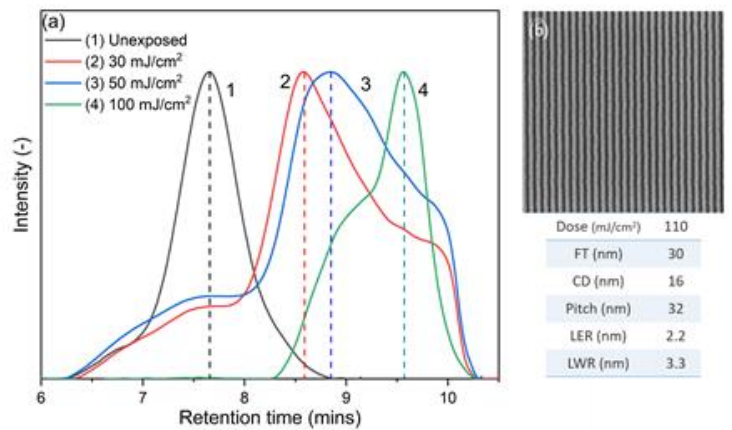

Figure 7 (a) Change in the Mw of the copolymer system 3 with exposure to different EUV-dose, (b) EUV-printability of the copolymer system at $16 \mathrm{~nm}$ dense $L / S$ patterns.

Therefore, as per the GPC results, the EUV-printability of this material is checked at a dose beyond $100 \mathrm{~mJ} / \mathrm{cm}^{2}$. It was confirmed that only by applying a EUV-dose beyond 100 $\mathrm{mJ} / \mathrm{cm}^{2}$, it was possible to resolve $16 \mathrm{~nm}$ dense patterns. Whereas, the lower doses could not resolve it. This confirms that the litho-parameters (peak shift and overlap) obtained from the GPC experiment can indeed be correlated with the EUV-printability of the material.

Furthermore, similar to PMMA, a bimodal/multimodal distribution is observed for the EUV-exposed copolymer system as well (in figure $7(a)$ ). And the amount of peak broadening is higher for the copolymer system as compared to PMMA. The reason for the peak broadening (in high $\mathrm{Mw}$ region) is studied by a Monte Carlo simulation tool called CHARIOT. ${ }^{23}$ Firstly, the amount of dose deposited in the resist film when a polymer system is exposed to an arbitrary EUV-dose is checked. The result is shown in figure $8(a)$, which suggests that in the case of EUV-exposure, the deposited dose is non-uniform throughout the film. That is, more dose is deposited on the top surface of the film as compared to the bottom interface. Please note that the deposited dose is zero at the top-most part of the film (i.e., at $\mathrm{FT}^{\sim} 35 \mathrm{~nm}$ ) because most of the secondary electrons generated in this area may either backscatter outside the film or pass into deeper sections of the film, thus reducing the overall deposited dose. Figure $8(\mathrm{~b})$ shows that most of the photoresist volume receives a nominal EUV-dose (indicated with a red dot), however, there is also a considerable amount of resist volume that receives lower than the nominal dose. Furthermore, when figure $8(b)$ is combined with the experimental values of change in $\mathrm{Mw}$ of copolymer system after EUV-exposure, and assuming a gaussian distribution of initial Mw material, figure 8(c) is obtained, which shows a tail of high $\mathrm{Mw}$ chains in the photoresist film, much like the one seen in the GPC results of the copolymer system (figure 7(a)). This tail of high $\mathrm{Mw}$ chains could be a potential cause of the problem in the EUV-printability of MCS-type photoresist platforms.
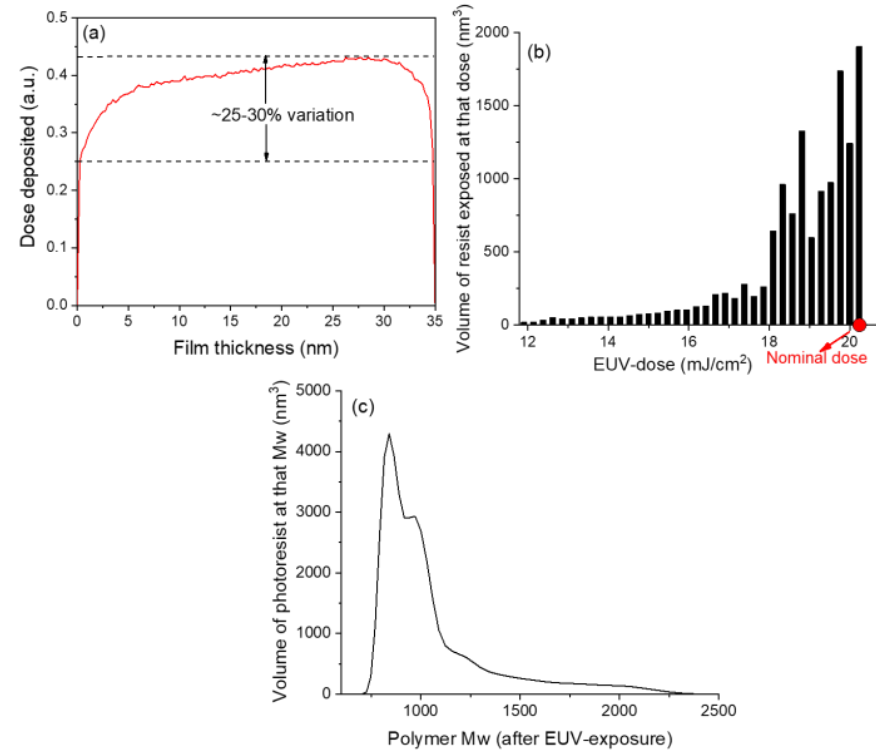

Figure 8 (a) Effective EUV-dose deposited through the resist film, when a photoresist system of arbitrary FT is exposed to an arbitrary EUV-dose, (b) The difference in EUV-dose received by the different volume of photoresist film) and (c) Tail of high Mw chains remaining in the MCS photoresist film after EUV-exposure. 
Furthermore, upon comparing the GPC results of PMMA and the copolymer system, it is seen that the broadening of the exposed peak is more exacerbated in the case of the copolymer system compared to the homopolymer system (PMMA). The reason behind this can be the complex chemistry and differences in the chain scission mechanism of the copolymer system. Also, the synthesis process of the copolymer system might play a role in this behavior. The effect of the copolymer synthesis process on the peak broadening and different $\mathrm{Mw}$ distribution through the photoresist film thickness is currently been undertaken and will be presented in future studies.

\subsection{Confirmation from the copolymer system}

The copolymer system validated the hypothesis that higher $\mathrm{Mw}$ material has a better EUV-printability than the lower $\mathrm{Mw}$ material. Furthermore, as seen from the GPC and EUVpatterning experiment, the copolymer system requires exposure with high EUV-dose $\left(>100 \mathrm{~mJ} / \mathrm{cm}^{2}\right)$ to pattern at 16 $\mathrm{nm} \mathrm{L} / \mathrm{S}$. Therefore, it might be necessary to include multiple high EUV-absorption groups to further increase its sensitivity. Chariot and GPC studies also suggest that the MCS-type photoresist might be hampered by the stochastics caused by their polymer properties and the non-uniform exposures caused by the EUV-light. Further studies need to be conducted to confirm this effect and to compare this with the stochastics observed in the CAR-photoresists.

\section{Conclusions}

This study was conducted to understand the effect of molecular weight on the scission mechanism of the main chain scission (MCS)-type photoresist platform for EUV lithography. Polymethylmethacrylate (PMMA) was selected as the model system on which the initial patterning and fundamental studies were carried on. Contrast curve analysis of PMMA showed that MIBK-IPA (1:3) developer solvent provides the best contrast with a EUV-dose-to-clear $\left(E_{0}\right)$ of $25 \mathrm{~mJ} / \mathrm{cm}^{2}$. Also, crosslinking in PMMA chains was observed at a high dose of $600 \mathrm{~mJ} / \mathrm{cm}^{2}$ and beyond. EUV-patterning experiment conducted on different Mw PMMA in Imec's ASML NXE3300B scanner showed that the higher $\mathrm{Mw}$ material has better performance than the lower $\mathrm{Mw}$ PMMA. Among all the different Mw PMMA, 950K Mw PMMA showed the best printability at $50 \mathrm{~nm}$ dense $\mathrm{L} / \mathrm{S}$ with the highest image contrast and the lowest LER of $4.1 \mathrm{~nm}$.

Fundamental tests were conducted to understand the effect of polymer properties on its printability. Firstly, EUV-induced desorption studies showed that the MCS-to-SCS ratio was proportional to the Mw. Additionally, LEE-induced desorption studies confirmed this effect and showed that the activation energy of PMMA lies at around $9 \mathrm{eV}$. Finally, GPC characterization of different Mw PMMA was used to analyze important litho-parameters, namely, peak-shift and peakoverlap. It was found that the higher Mw PMMA showed favorable changes in these litho-parameters compared to low Mw PMMA, which provided an explanation for their better EUVprintability.
The hypothesis obtained on a simple homopolymer system (PMMA) was validated with an engineered copolymer system. The patterning results confirmed that the highest Mw system shows the best EUV-printability. GPC analysis of one of the systems showed that it requires a high EUV-dose of $100 \mathrm{~mJ} / \mathrm{cm}^{2}$ to have a sufficient peak shift between the unexposed and the exposed material. Therefore, a EUV-dose of $110 \mathrm{~mJ} / \mathrm{cm}^{2}$ was applied to pattern $16 \mathrm{~nm}$ dense L/S with a low LER and LWR values of 2.2 and $3.3 \mathrm{~nm}$, respectively. GPC analysis of the copolymer system further showed peak broadening in the EUVexposed material, similar to PMMA, and the reason for that effect was studied by a Monte Carlo simulation tool, CHARIOT. It showed that the EUV-exposure is non-uniform throughout the resist film, which results in the bimodal/multimodal distribution of the exposed material and is not ideal for the litho-application. This effect was also seen in PMMA material but to a lesser extent, which might be because of its uniform homogenous chemistry. Whereas, peak broadening was exacerbated in the copolymer system due to its more complicated chemistry and scission-mechanism. Further studies are needed to understand this effect.

\section{Conflicts of interest}

There are no conflicts to declare.

\section{Acknowledgments}

The authors would like to thank Zeon corporation for providing the copolymer system for this study. This project has received funding from the European Union's Horizon 2020 research and innovation program under the Marie Skłodowska-Curie grant agreement No 722149.

\section{References}

1 Samsung Press Release, Samsung Electronics Starts Production of EUV-based 7nm LPP Process, Korea, 18/10/2018; Available at https://news.sam sung.com/global/ samsung-electronics-starts-production-of-euv-based-7nmIpp-process

2 T. Kozawa and S.Tagawa, Japanese Journal of Applied Physics, 2010, 49, 030001.

3 A. Saeki, T. Kozawa, Y. Ohnishi and S. Tagawa, The Journal of Physical Chemistry A, 2007, 111, 1229.

4 J. Torok, R. Re Del, H. Herbol, S. Das, I. Bocharova, A. Paolucci, L.E. Ocola, C. Ventrice Jr, E. Lifshin, G. Denbeaux and R.L. Brainard, Journal of Photopolymer Science and Technology, 2013, 26, 625-634.

5 A. Robinson and R. Lawson, Materials and Processes for Next Generation Lithography, 2011, Vol. 11, Elsevier.

6 D. De Simone, Y. Vesters and G. Vandenberghe, Advanced Optical Technologies, 2017, 6, 163.

7 T. Kozawa, J.J. Santillan and T. Itani, Japanese Journal of Applied Physics, 2013, 52, 076502.

8 P. De Bisschop, Journal of Micro/Nanolithography, MEMS, and MOEMS, 2017, 16, 41013.

9 P. De Bisschop and E. Hendrickx, International Society for Optics and Photonics IX, 2018, 10583, 105831K). 
10 J.W. Thackeray, Materials challenges for sub-20-nm lithography, Journal of Micro/Nanolithography, MEMS, and MOEMS, 2011, 10, 033009.

11 A. Narasimhan, S. Grzeskowiak, J. Ostrander, J. Schad, E. Rebeyev, M. Neisser, L.E. Ocola, G. Denbeaux and R.L. Brainard, Advances in Patterning Materials and Processes XXXIII, International Society for Optics and Photonics, 2016, 9779, 97790F.

12 A. Narasimhan, S. Grzeskowiak, C. Ackerman, T. Flynn, G. Denbeaux and R.L. Brainard, International Society for Optics and Photonics, 2017, 10143, 101430W.

13 R. Gronheid, T.R. Younkin, M.J. Leeson, C. Fonseca, J.S. Hooge, K. Nafus, J.J. Biafore and M.D. Smith, International Society for Optics and Photonics, 2011, 7969, 796904.

14 T. Kozawa, T. Shigaki, K. Okamoto, A. Saeki, S. Tagawa, T. Kai and T. Shimokawa, Journal of Vacuum Science \& Technology B: Microelectronics and Nanometer Structures Processing, Measurement, and Phenomena, 2006, 24, 3055.

15 H. Tsubaki, T. Yamanaka, F. Nishiyama and K. Shitabatake, Advances in Resist Materials and Processing Technology XXIV International Society for Optics and Photonics, 2007, 6519, 651918).

16 L. Li, X. Liu, S. Pal, S. Wang, C.K. Ober and E.P. Giannelis, Chemical Society Reviews, 2017, 46, 4855-4866.

17 K. Koshelev, M. A. Mohammad, T. Fito, K. L. Westra, S. K. Dew and M. Stepanovaa, J. Vac. Sci. Technol., 2011, 29, 06 F306.

18 A. Nakajima, T. Kozawa, M. Hoshino and M. Hashimoto, Proc. SPIE, 2018, 10810, 108100.

19 I. Pollentier, Y. Vesters, J. S. Petersen, P. Vanelderen, A Rathore, D. De Simone and G. Vandenberghe, Advances in Patterning Materials and Processes XXXV International Society for Optics and Photonics, 2018, 10586, 105860C.

20 NIST chemistry webbook [https://webbook.nist.gov/chemistry/].

21 I. Pollentier, G. Aksenov, A.M. Goethals, R. Gronheid, R. Jonckheere and M. Leeson, Alternative Lithographic Technologies, International Society for Optics and Photonics, 2009, 7271, 727146.

22 A. Striegel, W. Yau, J. Kirkland and D. Bly, Modern sizeexclusion liquid chromatography: practice of gel permeation and gel filtration chromatography, John Wiley \& Sons, 2009.

23 S. Babin, S.S. Borisov, H. Ito, A. Ivanchikov, D. Matison, V. Militsin and M. Suzuki, Scanning Microscopy, International Society for Optics and Photonics, 2010, 7729, 7290W.

24 S. Yasin, D.G. Hasko and H. Ahmed, Microelectronic engineering, 2002, 61, 745.

25 L. E. Ocola, M. Costales, M. and D.J. Gosztola, Nanotechnology, 2015, 27, 035302.

26 T. Fedynyshyn, R. Goodman, A. Cabral, C. S. Tarrio and T.B. Lucatorto, In Advances in Resist Materials and Processing Technology XXVII, SPIE, 2010, 7639, 76390A.

27 J. A. Moore, J.A. and Choi, J.O, Radiation Effects on Polymers, ACS Symposium Series, 1991, 475.

28 V. M. Bermudez, Journal of Vacuum Science \& Technology B: Microelectronics and Nanometer Structures Processing, Measurement, and Phenomena, 1999, 17, 2512.

29 J. Wang, C. Morin, L. Li, A. P. Hitchcock, A. Scholl, and A. Doran, Journal of Electron Spectroscopy and Related Phenomena, 2009, 170, 25.

30 D. Cloetta, P. Davor, Properties of La2-xSrxCuO4 under epitaxial strain: photoemission on ultra-thin films grown by pulsed laser deposition, 2005.

31 W. Sakai, A. Tsuchida, M. Yamamoto, J. Yamauchi, Journal of Polymer Science Part A: Polymer Chemistry, 1995, 33, 1969.

32 A. Schleunitz, V. A. Guzenko, A. Schander, M. Vogler and H. Schift, Journal of Vacuum Science \& Technology B, Nanotechnology and Microelectronics: Materials, Processing, Measurement, and Phenomena, 2011, 29, $06 \mathrm{~F} 302$.
33 H. Demers, N. Poirier-Demers, M. R. Phillips, N. De Jonge and D. Drouin, Microscopy and Microanalysis, 2012, 18, 1220.

34 J. Zhou, and X. Yang, Journal of Vacuum Science \& Technology B: Microelectronics and Nanometer Structures Processing, Measurement, and Phenomena, 2006, 24, 1202.

$35 \mathrm{H}$. Miyoshi and J. Taniguchi, Journal of Vacuum Science \& Technology B, Nanotechnology and Microelectronics: Materials, Processing, Measurement, and Phenomena, 2015, 33(6), 06FD05.

$36 \mathrm{H}$. Ikeura-Sekiguchi, T. Sekiguchi and M. Koike, Journal of electron spectroscopy and related phenomena, 2005, 144, 453-455. 